\title{
Green Materials: Referees 2020
}

The following is a list of referees who have reviewed papers for Green Materials between 1 January 2020 and 31 December 2020. The Institution of Civil Engineers is very grateful for their assistance.

We are continually looking for suitable reviewers for papers submitted to Green Materials. Papers published in ICE Science journals must be submitted to at least two independent referees to judge accuracy, style, impact, importance and interest.

Mohammad Al-Sayah

Raghu Anjanapura

Mohammad Aslam

Nurul Farhanah Azman

Shashwat Banerjee

Mateusz Barczewski

Mehul Barde

Justin Barone

Kiran Billa

Benoit Briou

Sylvain Caillol

Zeki Candan

Hassan Chaair

Caitlyn Clarkson

Hongxing Dai

Charles Daniels

Zoriana Demchuk

Gurjot Dhaliwal

Sylwia Dworakowska

Yunli Fang

Paulina Faria

Qinghua Feng

John Frostad
Miguel Gama

Kalyanrao Garadkar

Jennifer Garden

Vikas Gite

Etienne Grau

Stéphane Grelier

Erik Hagberg

Noury Hamdi

Dalila Hammiche

Zhe Han

Mohammad L. Hassan

Natalia Hernandez

Jinguang $\mathrm{Hu}$

Haw-Ming Huang

Chao Jia

Long Jiang

Raghava Reddy Kakarla

Senthil Kumar Kaliyavaradhan

Hamdy Khalil

Mohammad Reza Khosravani

Kerry Kirwan

Kazim Kose

Vincent Lapinte
If you are interested in reviewing articles on any topic related to green materials, please submit your name, qualifications or $\mathrm{CV}$, and areas of expertise. We are in need of individuals who will agree to review papers in a timely fashion (within 3 to 4 weeks of receipt) and provide confidential feedback to the Editorial Advisory Panel concerning the quality of the paper and any suggested revisions that would be appropriate.

If you are such a person, please contact Rebecca Rivers (tel.: +44 (0)207 665 2241; e-mail: rebecca.rivers@ice.org.uk) for more information on the referee process.

Changhe Li
Hongqiang Li
Zhiwei Li
Yanju Liu
Juan López-Martínez
Hongdian Lu
Dana Luca Motoc
Jyothi M. S.
Alejandro Manzano-Ramirez
Hafeezullah Memon
Selvaraj Mohana Roopan
Mina Mohebbi
Alex Morgan
Bahubali Murgunde
TriDung Ngo
Mohd Nor Faiz Norrrahim
Sasan Nouranian
Emeso Ojo
Mayuri P. V.
S. J. Pawar
Jesus Rodriguez-Nuñez
Jamie Rubinstein
Minoo Sadri

Kimihiro Sakagami

Olivier Sandre

Chinmay Satam

Carla Silva

Armando Jorge Silvestre

Zakia Sultana

Suresh Thangudu

Arun Torris

Ahmet Refah Torun

Prakash Wadgaonkar

Chaohui Wang

Rongpeng Wang

Xin Wang

Renate Maria Ramos Wellen

Ashok Kumar Yadav

Yuhaojie Yu

Mauro Zammarano

Kun Zang

Ali Zolfagharian 